\title{
PERFORMANCE EVALUATION OF NEMO IN SATELLITE NETWORKS
}

\author{
Abu Zafar M. Shahriar \\ Mohammed Atiquzzaman \\ School of Computer Science \\ University of Oklahoma, Norman, OK 73019. \\ \{shahriar, atiq\}@ou.edu
}

\author{
William Ivancic \\ Satellite Networks and Architectures Branch \\ NASA Glenn Research Center, Cleveland, OH 44135. \\ wivancic@grc.nasa.gov
}

\begin{abstract}
NEtwork MObility (NEMO) protocols can be used to manage aggregate mobility of multiple IP-enabled devices onboard a Low Earth Orbit satellite (a mobile network on-board). NEMO protocols enjoy several performance advantages, such as reduced signaling, increased manageability and conservation of satellite link bandwidth as compared to host mobility protocols for individual devices. In addition, NEMO protocols can provide continuous connectivity at upper layers using nested NEMO (a mobile network attached to another) during unavailability of ground stations where as connection would terminate if host mobility protocols were used. Therefore, NEMO protocols needs to be evaluated in space. We propose an architecture for NEMO in space, where the devices are connected together using an onboard Local Area Network. The architecture includes nesting where a mobile network on-board a satellite can attach to another. We evaluated NEMO protocols for the architecture using a space friendly data transfer protocol called Saratoga because widely used protocols like TCP is not space friendly. Simulation based performance evaluation shows continuity of connections at upper layers and performance superiority of Saratoga to TCP for NEMO in space.
\end{abstract}

\section{INTRODUCTION}

Spacecrafts and satellites contain devices to sense and to take measurements of Earth and space. To transfer data to IP-based terrestrial network, future Low Earth Orbit (LEO) satellites will contain multiple IP-enabled devices that are accessible through ground stations from the Earth. Constellations of LEO satellites like United Kingdom Disaster Monitoring Constellation (UK-DMC) [1] or future MILSATCOM [2] can be used to build information systems for commercial applications or to build intelligence, surveillance and reconnaissance systems for military applications. LEO satellites connects to different ground stations as they rotate around the Earth resulting in mobility of on-board devices that are connected to the Internet.

The research reported in this paper was funded by NASA Grant NNX06AE44G.

978-1-4244-2677-5/08/\$25.00 2008 IEEE
Mobility of devices while connected to the Internet is called host mobility that is not supported by location-based addressing scheme of the Internet. To allow host mobility, Internet Engineering Task Force (IETF) designed Mobile IP (MIP) [3] and MIPv6 [4]. MIP or MIPv6 suffers from limitations like handoff latency and inefficient routing that were addressed by a protocol called SIGMA [5] proposed by $\mathrm{Fu}$ et al.

Host mobility management protocols, like MIP or SIGMA, are not effective for managing the mobility of hosts that are moving together (in a vehicle or satellite containing IP-enabled devices) due to significant signaling overhead, increased power consumption and requirement for each host to have multiple powerful transceivers to communicate with access routers. Moreover, simple devices, incapable of running complex protocols (for example, MIP and SIGMA) due to limited resource and processing capability, are unable to communicate with the Internet.

To efficiently manage aggregate mobility, IETF has proposed NEtwork MObility (NEMO) where hosts that move together are connected in a Local Area Network (LAN), and a router in the LAN manages the mobility of all the hosts. This network in motion is called mobile network. A mobile network can attach to another mobile network to create a nested mobile network, and several levels of nesting can occur. Level of nesting affects the performance of NEMO Basic Support Protocol (NEMO BSP) [6], a logical extension of MIPv6, which performs better than MIPv6 for mobility management of a mobile network [7].

Future satellites will contain IP-enabled devices (such as camera, sensors, recording devices etc.) that are connected to terrestrial networks through ground stations. As the satellites connect to different ground stations during rotation, connections to on-board devices have to be handed off between ground stations. Managing the mobility of the devices in an aggregate fashion using NEMO can result in better utilization of satellite resources, such as limited device processing capability and on-board power 
availability. Moreover, upper layer connections to terrestrial networks are lost during handoff between ground stations due to unavailability of ground stations for a long period of time. Continuous upper layer connectivity can be achieved through other satellites using nested NEMO. Our objective is to investigate the applicability and performance of NEMO for mobility management of on-board devices and providing continuous connectivity to the devices during interruptions in satellite to ground connectivity.

National Aeronautics and Space Administration (NASA) has been experimenting with the use of Internet protocols in space [8]. Application of MIP to satellite networks has been proposed by Israel et al. [9] where an on-board device is considered a mobile host with mobility management agents residing in terrestrial networks. Leung et al. [10] presents the application of IPv4 based mobile network within a single satellite. Based on concepts similar to NEMO, Shi et al. [11] proposes a satellite constellation network architecture that allows communication of satellite hosts with terrestrial networks through satellite mobile routers while the relative movement of satellites are transparent to terrestrial networks. Our aim is to evaluate the application and performance of nested NEMO in satellite networks by ns-2 simulation.

Satellite links are asymmetric in capacity (high downlink and low uplink bandwidth) and lossy due to high error rate. Therefore, transport protocol like TCP is not suitable for data transfer in satellite networks because losses are misinterpreted as congestion loss resulting in reduced throughput. Moreover, TCP throughput, being proportional to acknowledgement receiving rate, is limited by the low uplink bandwidth. Saratoga, a file transfer protocol, overcomes the limitations of TCP and is currently used to download data from UK-DMC satellites operated by Surrey Satellite Technologies [1]. Saratoga sends data at a rate independent of acknowledgement rate; recovery of loss is based on periodic acknowledgements from receiver. We implemented Saratoga in ns-2, and compared nested NEMO in satellite networks with both TCP and Saratoga.

Our contributions in this paper are: (i) Application of NEMO to aggregate mobility management of on-board IPenabled devices in satellites, (ii) demonstration of continuous connectivity using nested NEMO despite interruption of connectivity of satellites with ground stations, (iii) performance comparison of NEMO in a satellite environment using TCP and Saratoga, and (iv) show how nesting affects determination of acknowledgement period of Saratoga. Simulation results show that continuous connectivity to terrestrial network can be ensured using nested NEMO despite that a ground station is unreachable. Performance comparison of Saratoga and TCP shows that Saratoga performs better than TCP specially when nesting occurs. In addition, results suggest that determining acknowledgement period in Saratoga needs to consider the effects of nesting to achieve better performance.

The rest of the paper is organized as follows. Sec. II presents the architecture and basic protocol of NEMO. Application of NEMO-based mobility management for satellite networks is presented in Sec. III. Sec. IV introduces Saratoga. Simulation results showing the performance of NEMO BSP in satellite networks are presented in Sec. V followed by conclusions in Sec. VI.

\section{NEMO}

In this section, we present the architecture and basic protocol of NEMO [6]. This will help the reader to understand the adaptation of NEMO to satellite networks in Sec. III.

\section{A. NEMO Architecture}

Fig. 1 shows the architecture of a mobile network to manage the mobility of the nodes as a network [6]. One or more routers called Mobile Router (MR) are employed to act as gateways for the nodes in the network. There could be different types of nodes inside the mobile network each called a Mobile Network Node (MNN). Different types of MNNs are - Local Fixed Node (LFN) that does not move with respect to the mobile network, Local Mobile Node (LMN) that usually resides in the mobile network and can move to other networks, Visiting Mobile Node (VMN) that get attached to the mobile network from the another network, and MR that can be an MNN to form a nested mobile network. A nested mobile network of multiple levels is formed when an MR attaches to another MR. In Fig. 1, MR1 is an MNN in MR's mobile network forming a nested mobile network. MRs attaches to the Internet through Access Routers (ARs). Whenever the point of attachment in the Internet is changed during movement, it is the responsibility of the MR to perform handoff and keep the transparency of the movement inside the mobile network.

The network to which a mobile network is usually connected is called the home network. An MR is registered with a router in its home network called Home Agent (HA) that performs location tracking and packet re-direction for MNNs. In Fig. 1 HA_MR and HA_MR1 are the HA for the mobile routers MR and MR1 respectively. Also, a node that communicates with MNNs is termed as Correspondent Node $(\mathrm{CN})$.

\section{B. NEMO Basic Support Protocol}

An MR registers with the HA and acquires a Home Address (HoA) through which it is reachable in the home network. MRs are also delegated one or more address 


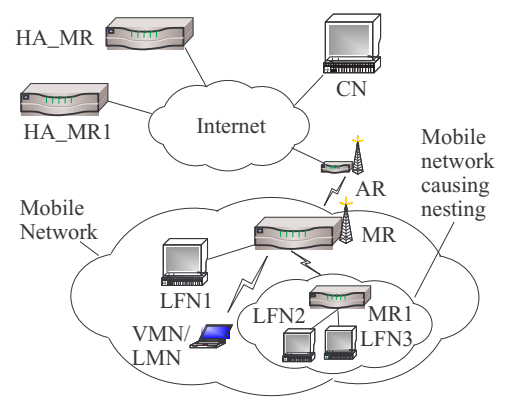

Fig. 1. Architecture of NEMO [6].

prefixes for use by MNNs inside its network. When the mobile network moves out of its home network to a foreign network, the MR obtains a new address called Care-ofAddress (CoA) from the foreign network and sends a Binding Update (BU) to its HA informing the new CoA. In addition to setting a bit in the $\mathrm{BU}$ to indicate that the MR is now acting as a router, it also contains the prefix of the mobile network. HA sends a positive Binding Acknowledgement (BA) to indicate that forwarding to the $M R$ is set and creates a binding cache entry that maps the HoA and prefixes of MR to the CoA of the MR. Once the binding process is completed, a bi-directional tunnel [12] is established between the HA and the MR, and HA tunnels all subsequent packets for the mobile network to the MR. As far as security is concerned, this binding process is secured by IPSec which is a required part in IPv6.

Fig. 2 shows the routing of packets for LFN1. When a $\mathrm{CN}$ sends a packet to a node in the mobile network, the packet is routed towards the HA, as HA advertises the prefix of MR in the network. The packet is then encapsulated and tunneled by the HA to the MR which receives, decapsulates and forwards the packet to LFN1. Packets in the reverse direction follow the reverse path undergoing encapsulation and decapsulation at MR and HA respectively.

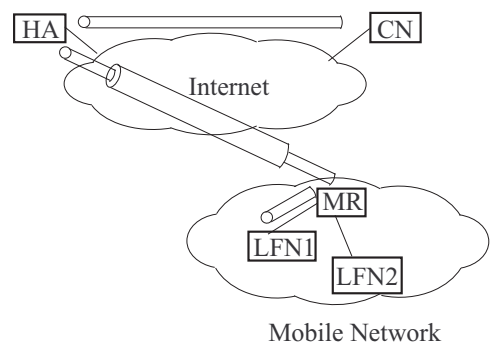

Fig. 2. Routing for LFN using bi-directional tunnel.

NEMO can be used to manage aggregate mobility of devices on-board a satellite. Sec. III presents the architecture of NEMO in satellite networks.

\section{NEMO IN SATELLITE}

In this section, we present an architecture to illustrate the application of NEMO to satellite networks, and show use of nested NEMO can achieve continuous connectivity.

\section{A. Basic NEMO in satellite network}

Fig. 3 shows the architecture for NEMO in satellite. Satellites, that are connected to ground stations (co-located with ARs) through Ground to Satellite Links (GSLs), handoff between ARs during movement around the Earth. These satellites carry on-board equipment for data collection. Since satellites handoff between ARs, these IP-enabled equipment can be considered as mobile nodes in space. If the on-board equipment (e.g. LFN1, LFN2 in Fig. 3) are connected to a Local Area Network with an MR on-board a satellite, the mobility of the nodes can be managed in an aggregated fashion by considering the LAN as a mobile network and managing the mobility of the LAN (in contrast to individual nodes as in Mobile IP) by the MR. Other key features of the architecture are as follows:

- Home network of the mobile network is in the terrestrial network where the HA_MR resides.

- MR communicates with the HA_MR over the satellite links.

- Mobile network in the satellite is handed off between access routers AR1 and AR2. Access routers are colocated with ground stations on the Earth.

- $\mathrm{CN}$ can be any node in terrestrial network that is downloading data from on-board devices.

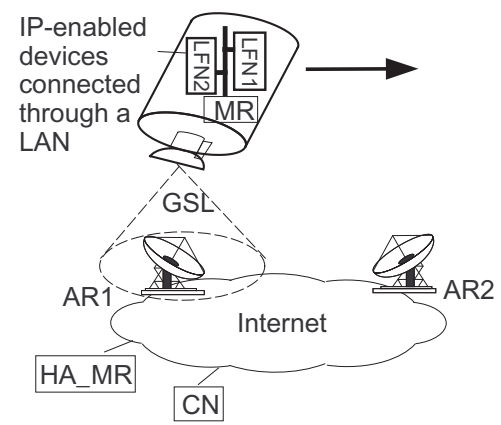

Fig. 3. Architecture of NEMO in satellite.

NEMO BSP (described in Sec. II-B) can be used to handle mobility of this mobile network in the satellite. In this mobile network, we only consider LFNs as it is not practical to have LMNs or VMNs inside a satellite. Since ARs co-located with ground stations are unavailable for a long period of time, delay in handoff between ARs will be large resulting in no connectivity to terrestrial network for a long time. To provide connectivity during handoff between ARs, nested NEMO can be used that allows an on-board MR to communicate with ARs through MRs on-board other satellites.

\section{B. Nested NEMO in satellite network}

An MR on-board a satellite, unable to find a ground station within its range, can handoff to an $M R$ in another 
satellite having connectivity to a ground station. MRs onboard different satellites (in a constellation proposed in [2]) connect through Inter Satellite Links (ISLs). In Fig. 4, MR1 looses connection with AR1 and hands off to MR2 which is connected to AR2. Thus MR1 becomes nested under the mobile network of MR2 and maintains its connectivity to terrestrial networks. An MR can connect to the terrestrial network through multiple MRs, creating multiple levels of nesting. Since satellites connects to ground stations for a brief period of time, nested NEMO can provide continuous IP connectivity to terrestrial networks.

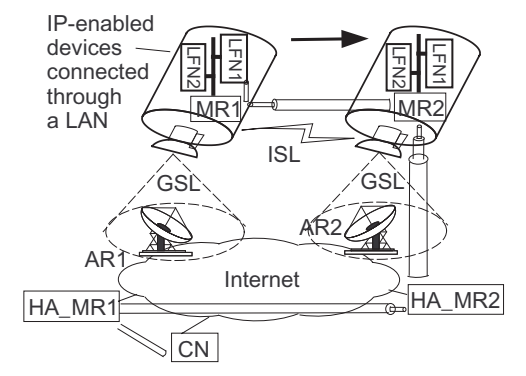

Fig. 4. Nested NEMO in satellite networks.

Performance evaluation of NEMO in satellite networks requires data transfer from LFNs to $\mathrm{CN}$. The protocol that we used for data transfer from LFNs to $\mathrm{CN}$ is introduced in Sec. IV.

\section{DATA TRANSFER PROTOCOL FOR SATELLITE NETWORKS}

Usually devices (LFNs) collect data that are downloaded by CNs in the terrestrial networks. TCP, which is a widely used transport protocol in terrestrial network, is not suitable for satellite networks because of the following characteristics of satellite links:

- High loss rate which is due to link errors but not due to congestion.

- Brief period of connectivity with ground stations resulting in discontinuity in IP connectivity.

- High asymmetry of uplink/downlink (high downlink and low uplink).

TCP has the following limitations when used over satellite links:

- TCP is designed for terrestrial networks where losses occur mainly due to congestion that can be overcome by reducing data sending rate. Therefore, TCP misinterprets high loss in satellite links as indication of congestion, and thereby unnecessarily reduces data sending rate.

- TCP connections are lost due to large handoff delays when satellites handoff between ground stations (ARs).
- TCP throughput depends on the acknowledgement receive rate from receiver. Low uplink limits acknowledgement rate resulting in low throughput when downloading data from LFNs to $\mathrm{CN}$.

To overcome the limitations of TCP over satellite links, Saratoga is being used as a file transfer protocol to download data from satellites such as the UK-DMC [1]. Saratoga is intended for efficient use of one hop highly asymmetric links having brief period of connectivity and has the following characteristics:

- It uses UDP as underlying transport protocol.

- Considering brief periods of connectivity of satellites to ground stations, it tries to send as much data as possible when the link is connected. New version of Saratoga has the provision of congestion control if sufficient feedback path is available.

- It uses less acknowledgement information for loss recovery considering low uplink capacity.

- Capable of resumption of data transfer from the position where it was left off.

Saratoga is based on sending packets at a rate independent of receiving acknowledgement from receiver. Periodically sender requests for acknowledgement from receiver. Receiver responds by sending acknowledgement that reports lost data. Sender retransmits lost data, and does not transmit any new data until all lost data are retransmitted.

For Saratoga to achieve optimal performance, it is very important to to set the interval of sending acknowledgements, which depends on uplink capacity. Too small interval will result in bottleneck at uplink similar to what happens for TCP. Too large an interval means lack of synchronization between sender and receiver for a long time, and requires large buffer at receiver for partially received data. However, finding interval of acknowledgement to optimize performance of Saratoga is out of scope of this paper. We show (see Sec. V-B2) that duplicate retransmission of packets occurs when interval of acknowledgement is small compared to volume of lost data and Round Trip Time (RTT). Number of duplicate retransmitted packets increases with the increase in number of hops between an MR and a ground station due to increased RTT. Duplicate packets, which consume bandwidth, are discarded by receiver resulting in reduced throughput of useful data. Moreover, number of hops between an MR and a ground station depends on level of nesting in NEMO. Therefore, setting the value for interval of acknowledgement should take into account the effect of nesting in NEMO.

To find how Saratoga performs with NEMO BSP, we used ns-2 simulation as discussed in Sec. V. 


\section{Performance evaluation}

This section presents the simulation topology and analysis of the results obtained from simulation.

\section{A. Topolgy}

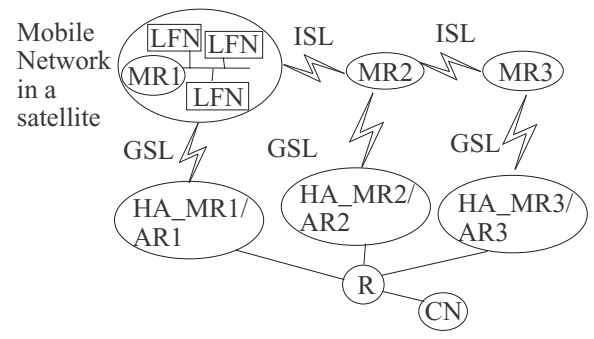

Fig. 5. Simulation topology for NEMO in satellite.

Fig. 5 shows the simulation topology. Initially MR1, MR2 and MR3 are in home network, placed consecutively in a plane in the orbit, and only MR1 contains a mobile network. Consecutive MRs are connected through ISLs while each is connected to the AR (ground station) through GSL at the same time. We put HAs collocated with ARs for convenience; this does not make any difference in the performance if we had put them separate. LFNs are data senders and $\mathrm{CN}$ is the receiver. Values of parameters used in the simulation are given in Table I. We simulate the following handoff scenarios to demonstrate multiple levels of nesting and an handoff to ground station when one becomes available:

- MR1 loose connection with AR1 and hands off to MR2.

- MR2 loose connection to AR2 and hands off to MR3.

- MR1 hands off to AR2.

Positions of ground stations and satellites were chosen to demonstrate the above mentioned handoff scenario. The values for other parameters are the same as in [11]. Downlink/uplink capacities are set to $8.134 / 0.0384 \mathrm{Mbps}$ as is currently being used or expected to be used by UK-DMC [1].

\section{B. Results}

We measured throughput, uplink utilization and RTT to evaluate and compare the performance of TCP and Saratoga over NEMO BSP. Throughput was measured as a function of time to find the effect of nesting caused by different handoff scenarios (Sec. V-A). RTT was measured as a function of time to show its effect on throughput. Uplink utilization explains the differences in achieved throughput for TCP and Saratoga. Duplicate packet retransmission is measured to show how number of hops between an MR and a ground station (AR) that results from nesting affects performance of Saratoga. Analysis of the results are presented in the following subsections.
TABLE I

VALUES OF PARAMETERS USED IN THE SIMULATION.

\begin{tabular}{|l|l|}
\hline Altitude & $780 \mathrm{~km}$ \\
\hline Orbital inclination & 86.4 \\
\hline Elevation mask & 8.2 \\
\hline Ground station (HA_MR1/ AR1) & $(0,110.0)$ \\
\hline Ground station (HA_MR2/ AR2) & $(42.0,112.0)$ \\
\hline Ground station (HA_MR3/ AR3) & $(70.0,125.0)$ \\
\hline MR1 & $(14.0,110.0)$ \\
\hline MR2 & $(55.0,110.0)$ \\
\hline MR3 & $(60.0,110.0)$ \\
\hline Satellite downlink & $8.134 \mathrm{Mbps}$ \\
\hline Satellite uplink & $0.0384 \mathrm{Mbps}$ \\
\hline Ethernet capacity & $100 \mathrm{Mbps}$ \\
\hline Ethernet link delay & $1 \mathrm{~ms}$ \\
\hline Terrestrial link capacity & $10 \mathrm{Mbps}$ \\
\hline Terrestrial link delay & $1.8 \mathrm{~ms}$ \\
\hline Data sending rate for Saratoga & $8 \mathrm{Mbps}$ \\
\hline Interval of acknowledgement & $4 \mathrm{~s}$ \\
\hline
\end{tabular}

1) Throughput: Throughput is measured by the amount of data received per second at $\mathrm{CN}$ from all LFNs. Fig. 6 shows the throughput of TCP and Saratoga for different number of LFNs. We present the salient features of Fig. 6 as follows:

- Continuous connectivity: As can be seen from throughput level, LFNs' connections to CN above IP layer is not terminated when the ground station is unavailable (in Region2, Region3). Temporary disruption in data transfer occurs (fall of throughput) due to handoff latency.

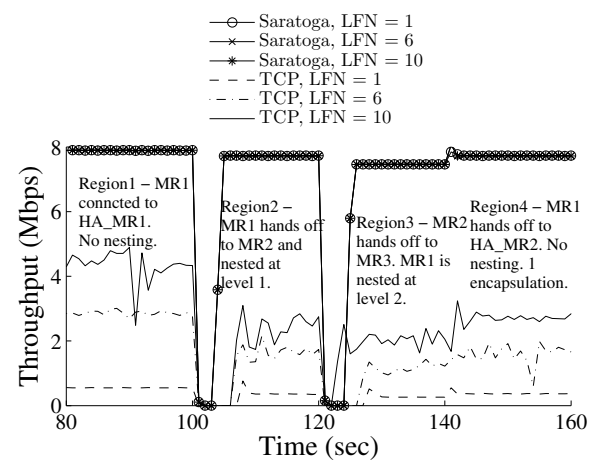

Fig. 6. Throughput at $\mathrm{CN}$ for different transport protocols.

- Throughput for Saratoga is higher than TCP: Throughput for Saratoga is higher than that of TCP due to low uplink capacity. Saratoga senders at LFNs send data at a rate close to downlink capacity (much higher than uplink), and is independent of rate of receiving acknowledgements from receiver $(\mathrm{CN})$. Therefore, throughput is close to downlink capacity. On the other hand, throughput of TCP is directly proportional to rate of receiving acknowledgements from $\mathrm{CN}$. Low uplink capacity limits the acknowledgement rate, and hence the throughput for TCP. 
Uplink utilization for Saratoga and TCP can be observed from Fig. 7. Uplink utilization is measured by number of acknowledgement packets per second. It can be seen that uplink is almost saturated for TCP whereas it is barely utilized for Saratoga.

- Differences in throughput for both Saratoga and TCP (Fig. 6): There are four regions based on the level of throughput as follows:

- Region1: In this region, MR1 is connected to HA_MR1 (AR1) in home network with one hop between MR1 and AR1 (no nesting). Maximum possible throughput is achieved in this region.

- Region2: MR1 hands off to MR2 which is in its home network. Thus MR1 is nested at level one with two hops between MR1 and AR2. Being inversely proportional to RTT, TCP throughput reduces in this region because of increased RTT (see Fig. 8). Large propagation delay in the ISL between MR1 and MR2 (due to large distance) contributes mostly to the increased RTT. Transmission time of additional header due to encapsulation of packets also contributes to the increased RTT. Small decrease in throughput results from additional header from encapsulation. Throughput for Saratoga decreases only due to additional header from encapsulation.

- Region3: MR2 hands off to MR3 while MR1 is still connected to MR2. Therefore, MR1 is nested at level two with three hops between MR1 and AR3. Reason for decrease in throughput is the same as that of Region2.

- Region4: MR1 hands off to HA_MR2 (AR2) reducing hop count to one (no nesting). Reduction of throughput in this region compared to throughput of Region1 (where MR1 was in home network) is due to larger RTT (from traversing HA_MR1 and additional encapsulation header) and additional header from encapsulation.

- Differences in TCP throughput for different number of LFNs: Due to increased data resulting from increased number of TCP senders, TCP throughput increases with increased number of LFNs. Increase of data sending rate from TCP senders can be observed in Fig. 9, which shows data sent by all TCP senders. For Saratoga, total data sending rate by all LFNs were always kept close to downlink rate to maximize link utilization.

2) Duplicate retransmission in Saratoga: Duplicate packet retransmission phenomena in Saratoga is delineated in Fig. 10. Duplicate retransmission occurs when acknowledgement comes to sender while the sender is

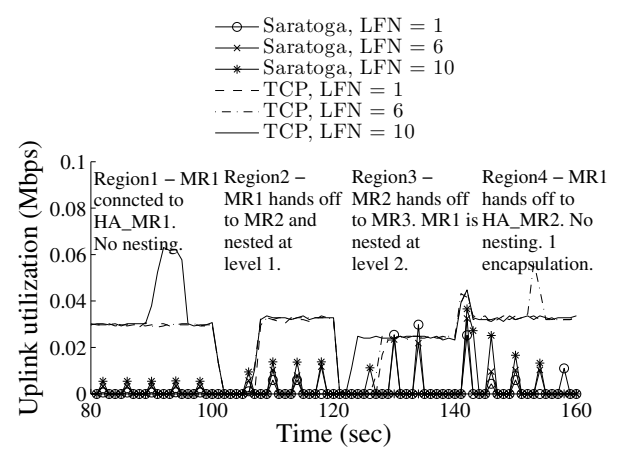

Fig. 7. Uplink utilization for Saratoga and TCP.

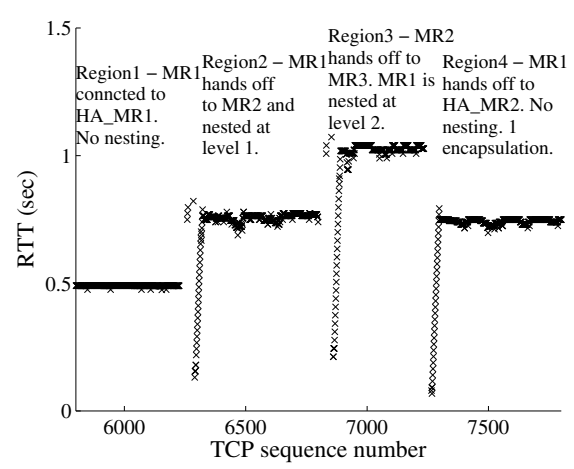

Fig. 8. A snapshot of RTT vs time for TCP and ATCP.

retransmitting. This can happen for two reasons. One is large retransmission time (due to large number of lost packets) compared to interval of acknowledgement. Other one is interval of acknowledgement small compared to RTT (time between sending a request for acknowledgement and receiving an acknowledgement). Here, we consider only the effect of RTT.

Fig. 11 shows the effect of duplicate packet retransmission for different hop counts between MR1 and a ground station (AR). Large RTT reduces the time between receiving acknowledgement (that trigger retransmission) and sending request for next acknowledgement (see Fig. 10). As a result, it can happen that sender sends an acknowledgement request to receiver while retransmitting. Therefore, packets that are retransmitted after sending acknowledgement request are received at receiver after the acknowledgement

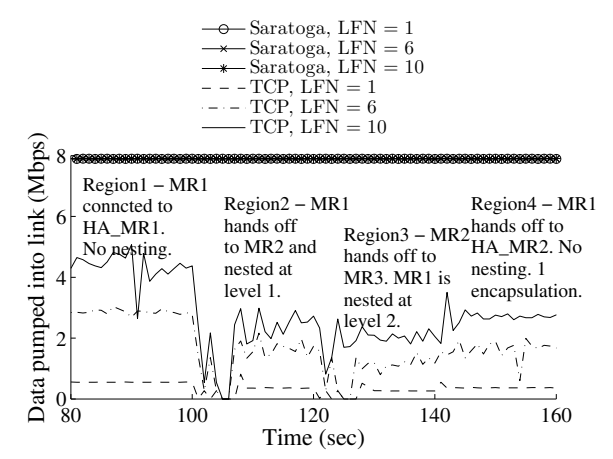

Fig. 9. Data sent by Saratoga and TCP senders for different number of LFNs. 
request. Hence, the packets, that are retransmitted during RTT period, are again reported as lost. These packets that are already received at the receiver after sending the acknowledgement are retransmitted again and are discarded at receiver. Moreover, the larger the RTT the larger the number of retransmitted packets during RTT, resulting in larger number of duplicate retransmitted packets. Since RTT increases with increased hop count due to nesting, number of duplicate retransmitted packets also increases with nesting level.

Increasing interval of acknowledgement sending reduces the number of packets that are retransmitted after sending the acknowledgement request. Therefore, number of duplicate retransmitted packets decreases with increased acknowledgement sending interval.

Duplicate retransmission occurs after handoff because large amount of packets are lost during handoff resulting in large retransmission time compared to acknowledgement sending interval and RTT. That is why there were no duplicate retransmission in Region1.

Results suggests that determining acknowledgement sending interval should consider the effect of nesting in NEMO.

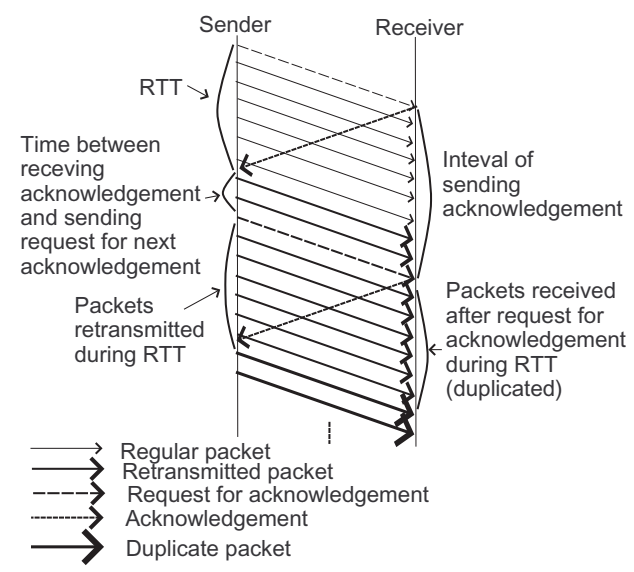

Fig. 10. Duplicate retransmission of packets in Saratoga.

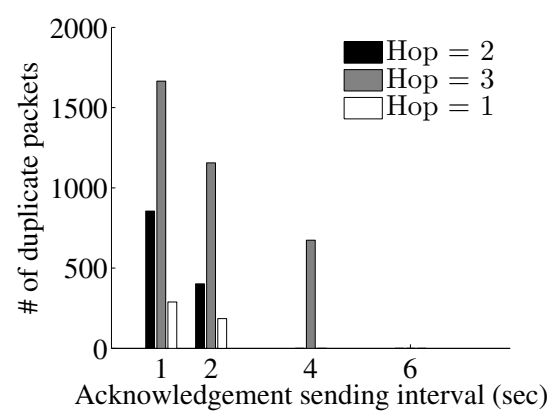

Fig. 11. Duplicate packet count for different number of hops between MR1 and the ground station.

\section{CONCLUSION}

In this paper, we study the effectiveness and performance of NEMO BSP for mobility management of devices on- board a satellite. Results show that continuous connectivity to devices on-board a satellite can be provided using nested NEMO despite loss of direct connection with ground stations. We used a space friendly file transfer protocol called Saratoga for transferring data from devices on-board a satellite to ground. Performance comparison of Saratoga with TCP shows that Saratoga performs better than TCP in space environment. Specially, performance of TCP drops when nesting occurs, while performance of Saratoga is not affected. We also reveal that configuring Saratoga for better performance needs to consider effects of nesting in NEMO.

However, to apply NEMO for classified applications (i.e. military intelligence, surveillance and reconnaissance), security measures to be incorporated. Since MNNs are unaware of mobility, security check points need to be enforced at MRs and HAs, possibly, by using IPSec to encrypt tunneled packets. Research on NEMO security is in its incipient stage and needs to be investigated further before it can be useful for classified applications.

\section{REFERENCES}

[1] L. Wood, "Saratoga a bundle convergence layer," Presentation, Delay-Tolerant Networking session IETF 69, Chicago, July 2007.

[2] R. Landry, D. Scher, J. Burdin, and P. Chen, "An efficient packet switching architecture for next-generation military satellite networks," in IEEE Military Communications Conference, Oct 13-16 2003, pp. 268-273.

[3] C. Perkins, "IP mobility support for IPv4," RFC 3220, January 2002.

[4] D. B. Johnson, C. E. Parkins, and J. Arkko, "Mobility support in IPv6," RFC 3775, June 2004.

[5] S. Fu and M. Atiquzzaman, "SIGMA: A transport layer handover protocol for mobile terrestrial and space networks," in e-Business and Telecommunincation Networks, J. Ascenso, L. Vasiu, and C. Belo, Eds. Springer, 2006, pp. 41-52.

[6] V. Devarapalli, R. Wakikawa, A. Petrescu, and P. Thubert, "NEtwork MObility (NEMO) basic support protocol," RFC 3963, January 2005.

[7] A. Z. M. Shahriar and M. Atiquzzaman, "Network mobility in satellite networks," in NASA Science Technology Conference, College Park, MD, June 19-21, 2007.

[8] W. Ivancic, P. Paulsen, D. Stewart, D. Shell, and L. Wood, "Secure, network-centric operations of a space-based asset - an abridged report," in Earth-Sun System Technology Conference, College Park, MD, June 28-30, 2005.

[9] D. Israel, R. Parise, K. Hogie, and E. Criscuolo, "Space communication demonstration using Internet technology," http://ipinspace.gsfc.nasa.gov/documents/ITC02-CANDOS.doc, 2002.

[10] K. Leung, D. Shell, W. D. Ivancic, D. H. Stewart, T. L. Bell, and B. A. Kachmar, "Application of Mobile-IP to space and aeronautical networks," IEEE Aerospace and Electronic Systems Magazine, vol. 16, no. 12, pp. 13-18, 2001.

[11] D. Shi and C. Tang, "The handoff study on satellite networks based on mobility network," in 6th International Conference on ITS Telecommunications, Chengdu, China, June 21-23, 2006, pp. 1029-1032.

[12] A. Conta and S. Deering, "Generic packet tunneling in IPv6 specifications,” RFC 2473, December 1998. 